\title{
Heart rate events classification via explainable fuzzy logic systems
}

\author{
Anis Jannah Dahalan, Tajul Rosli Razak, Mohammad Hafiz Ismail, Shukor Sanim Mohd Fauzi, Ray \\ Adderley JM Gining \\ Faculty of Computer and Mathematical Science, Universiti Teknologi MARA, Arau, Malaysia
}

\begin{tabular}{|c|c|}
\hline Article Info & ABSTRACT \\
\hline Article history: & As people, we have no way of knowing whether our heart rate is considered \\
\hline Received Jun 9, 2021 & normal or not. The strength and quality of our pulses will deteriorate as we get \\
\hline Revised Aug 15, 2021 & requires immediate attention. The main goal of this paper is to define the heart \\
\hline Accepted Aug 27, 2021 & $\begin{array}{l}\text { rate stage using fuzzy logic systems (FLSs). In practice, however, designing } \\
\text { or developing fuzzy logic systems is extremely difficult. To achieve this aim, }\end{array}$ \\
\hline Keywords: & $\begin{array}{l}\text { we suggested a solution that involves: i) classifying the medical expert's } \\
\text { criterion for signs of heart rate; ii) developing an explainable fuzzy logic }\end{array}$ \\
\hline Explainable system & system for heart rate measurement; and iii) evaluating the proposed system \\
\hline Fuzzy logic system & $\begin{array}{l}\text { With human experts. In adition, the aim of this research was to provide an } \\
\text { explainable fuzzy system that people could use to self-monitor heart rate }\end{array}$ \\
\hline Heart rate classification & levels and determine their health status. As a result, it is hoped that this \\
\hline Medical application & $\begin{array}{l}\text { research would provide insight into how to improve the development of fuzzy } \\
\text { logic systems, especially in the field of medical applications. }\end{array}$ \\
\hline
\end{tabular}

This is an open access article under the CC BY-SA license.

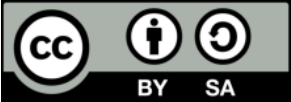

\section{Corresponding Author:}

Tajul Rosli Razak

Faculty of Computer and Mathematical Science

Universiti Teknologi MARA

Perlis Branch, Arau Campus, 02600 Arau, Perlis, Malaysia

Email: tajulrosli@uitm.edu.my

\section{INTRODUCTION}

A heart rate (HR) is a simple vital sign that can be used to determine prognosis [1]. Simply put, the human body's heart rate is an effective health predictor [2]. The heart rate can be measured in a variety of places on the human body, including the shoulders, elbows, the top of the foot, and the side of the spine, according to the American Heart Association [3]. Heart rate cases include tachycardia, atrial fibrillation, atrial flutter, bradycardia, ventricular fibrillation, and premature contractions. In this paper, this investigation would concentrate only on cases of tachycardia and bradycardia.

During exercise or as a physiological reaction to stress, trauma, or sickness, a person's heart rate will naturally increase [4]. And while sleeping, a person's heart can pound quicker than usual, which can be a concern. It's referred to as 'tachycardia' in psychiatric terminology. What is considered too young, though, is dependent on age and general health [5]. An underlying health condition, anxiety or stress, tiredness, excessive caffeine intake, heavy alcohol consumption, and excess electrolytes may all contribute to tachycardia. Tachycardia may also be caused by an underlying medical condition like anaemia, congenital heart disease, heart disease with low blood pressure, hyperthyroidism, or heart injury. This pathological state of the heart rate may be temporary.

In the medical field, fuzzy systems are widely used. As discussed in [6], fuzzy logic is a method of computation that focuses on "degrees of truth" rather than the traditional "true or false" (1 or 0$)$ for modern 
machines relying on boolean logic. Fuzzy logic is intended to resolve problems by taking into account all available data. As a result, depending on suggestions, the appropriate decisions can be taken. Furthermore, any ambiguity can be easily handled by using fuzzy logic [7]. The pillar of human speech is also the base of fuzzy logic. This is because fuzzy logic is founded on the mechanisms used for qualitative definition in daily speech. Therefore, fuzzy systems seems to be a key to explainable artificial intelligence (XAI) [8].

However, since medical applications have too many complex variables, developing a fuzzy logic system in practice is challenging [9]. To begin, we present a mechanism for building an explainable fuzzy logic system for classifying heart rate events such as tachycardia, normal, and bradycardia in this paper. This fuzzy logic system is intended to detect any potential health issues that can arise as a result of the event. To do so, we present in this paper the method for developing this explainable fuzzy logic system, which involves: i) medical expert acquisition of heart rate; ii) step-by-steps in constructing fuzzy logic systems; iii) demonstration of fuzzy systems-GUI; and iv) evaluating the suggested solution.

\section{BACKGROUND} section.

We provide some background on heart rate, classification systems, and fuzzy logic systems in this

\subsection{Heart rate}

According to an analysis in [10], the HR is defined as the number of heart beats per minute. Heart rate is affected by several factors. Just a few examples include air temperature, body posture, emotions, body height, and drug use. All areas of the body are unable to calculate the heart rate. The most often hurt body parts are the wrists, inside elbow, side of the thigh, and top of the foot. This paper only discusses major events that cause heart rates, such as tachycardia and bradycardia.

A heart rate of less than 60 beats per minute is called bradycardia [11]. For certain people, a sluggish heart rate may be physiologically normal, and for others, heart rates of 60 beats per minute may be insufficient, as shown in Figure 1. Damage to heart tissue caused by ageing is one of the causes of bradycardia, which can lead to heart disease or a heart attack. Such conditions include a congenital heart defect, inflammation of heart tissue, a complication of heart surgery, among others.

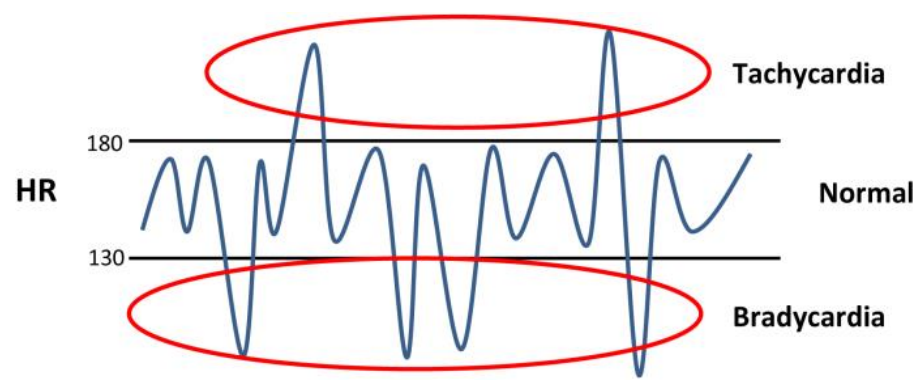

Figure 1. Heart rate classification (bpm-beat per minutes)

Tachycardia, which is characterized as an atrial or ventricular rate of more than 100 beats per minute and may be physiological or pathological, is a frequent phenomenon [12]. At these levels, the heart is unable to efficiently pump oxygen-rich blood to the body. The two types of tachycardia are atrial tachycardia (upper heart chambers) and ventricular tachycardia (lower heart chambers) (ventricular tachycardia). Tachycardia may be caused by heart-related conditions such as high blood pressure (hypertension), emotional fatigue, or over intake of alcoholic or caffeinated beverages, among others.

\subsection{Classification systems}

The classification system involves categorizing and arranging lists of objects in a rational and useful manner. Additionally, any collection of objects may have several useful classification systems. Linnaean taxonomy (classifying living things) and Dewey decimal classification are two examples of well-known classification schemes (classifying non-fiction books). In this paper, a few classification algorithms are discussed in the following section.

An artificial neural network (ANN) is a kind of computer that uses artificial intelligence. It is focused on studies in living organisms' brains and nervous systems [13]. It is proper to fix the problem. ANN 
provide the potential to learn and model nonlinear and complex relationships. Menwhile, a well-known tool for a variety of data mining operations is the support vector machine (SVM). Outliner understanding, regression, and classification are also part of this. SVM [14] is a set of supervised learning approaches for classification and regression that are identical. SVM employs mathematical learning theory to discover a regularized hypothesis that closely fits the available evidence while avoiding overfitting. The decision tree is a tree structure that looks like a flowchart [15]. A test on an attribute is represented by each internal node in the decision tree. Each mark represents a test result, and each leaf node represents a class code. A decision tree can easily be converted into classification criteria.x.

\subsection{Fuzzy logic systems}

Since the class with the highest similarity [16], fuzzy logic is clear to grasp. The fuzzy logic algorithm can also be represented with little details. Aside from that, fuzzy logic will operate with any kind of input, whether it is imprecise, warped, or noisy. As shown in Figure 2, fuzzification, fuzzy inference, and defuzzification are central components of a fuzzy logic system.

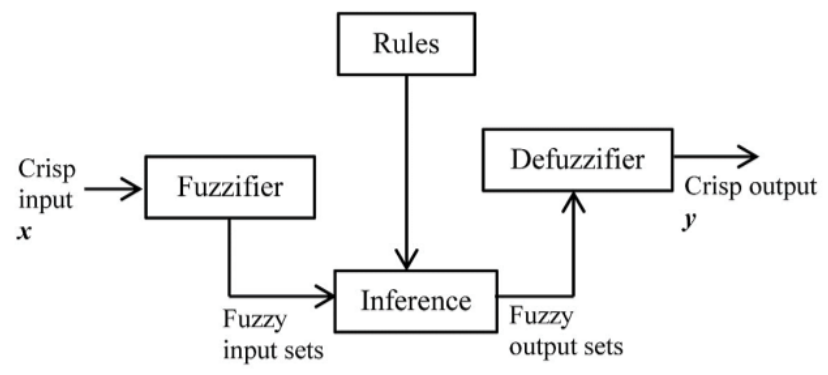

Figure 2. Fuzzy logic system. Adapted from [7]

The following are the major phases in the FLS (fuzzy logic system) as shown in Figure 2: i) the fuzzification component converts each crisp input variable into a membership grade based on the membership functions defined; ii) the fuzzy reasoning method is then carried out by the inference engine, which employs the necessary fuzzy operators to produce the fuzzy collection that will be accumulated in the output variable; and iii) the defuzzification component uses a specific defuzzification process to produce the fuzzy collection that will be accumulated in the output variable. One of the most compelling reasons to use FLSs for machine modelling is that they use easy-to-understand linguistic variables and rules [8]. Furthermore, thanks to their linguistic modelling and estimated thinking skills, FLSs are good at capturing the scope of a wide variety of problems [17].

\subsection{Fuzzy logic systems on medical application}

Fuzzy logic plays an important part in some fields of medicine. Breast cancer [18], heart disease [19], [20], lung cancer [21], [22], liver [23], [24], and diabetes [25], [26] are only a few of the medical realms where fuzzy logic systems (FLSs) have been effectively implemented. In addition, the FLSs is useful for objective research in medical diagnosis $\backslash$ cite $\{$ greeda2018study\}. In the medical field, fuzzy expert systems are used to learn knowledge, cope with inconsistencies, schedule care, provide advice, track and manage the structure, forecast parameters, and think artificially. The methodology used is presented along with the fundamentals of fuzzy logic and its application fields in the medical domain.

\section{MEDICAL EXPERT ACQUISITION ON HEART RATE CLASSIFICATION}

In FLS, fuzzy rules have been used as a primary way to express knowledge since they are more suitable and flexible than traditional IF-THEN rules. However, building a rule base, especially from human experts, is one of the most challenging tasks for FLSs [27]. This is due to the lack of a consistent system for designing fuzzy rules, especially where human experts are used. This section discusses the method for producing fuzzy logic rules from human experts, especially medical experts.

In this study, a mixed-mode approach, consisting of an interview and a survey, was used to gather actual information. This research includes an interview session with three medical experts. The survey on heart rate is then performed with medical experts. 


\subsection{Interview}

The first medical expert is Dr. A, a medical officer. The second medical expert is Mrs B, a pharmacist. The third medical expert is Dr. C, a medical officer. All three medical experts are from Hospital Sultanah Maliha, Langkawi, Kedah. During a conversation with Dr. A, it was found that there was no difference in heart rate between men and women. To put it another way, all men and women have the same heart rate. During an interview with Mrs B, some symptoms of tachycardia and bradycardia were found. Intermittent heartbeats, blurred vision, palpitations, and chest pain are also symptoms of tachycardia. Fatigue, dizziness, and shortness of breath are all symptoms of bradycardia. In other words, there is a difference in signs between tachycardia and bradycardia. Finally, it was found during the consultation with Dr. C that the paediatrics group had a higher heart rate than the infants. In other words, the heart rhythms of children were lower than those of the public.

\subsection{Survey}

During this process, medical experts performed a heart rate survey. Five medical practitioners took part in the study. It's worth noting that three of the five medical professionals who took part in the interview was the same person. There are 36 questions in this survey. The results of this survey will be used to build the MATLAB rule base for this scheme. For example, in this survey, question 3 is depicted in Figure 3.

\section{If heart rate is slow and age is young and fitness level is above average?}

5 responses

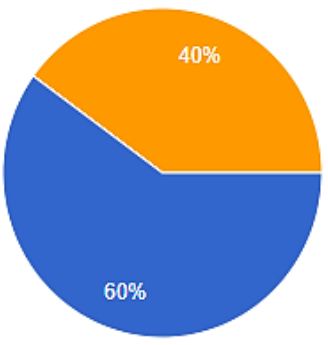

Figure 3. What would happen if the heart rate were slow, the age is young, and the fitness level is above average?

\subsection{Capturing the rules from the experts}

The aim of this section is to look at how to assess the rule base by looking at the survey results (from the previous section). Each survey query has the goal of obtaining the rule base for this study. We used the plurality of expert responses for each question to determine the result for each guideline.

Figure 4 shows an example of question 1 from this survey. We may always receive a straightforward answer in order to obtain the result of the rules as shown in Figure 3. However, as shown in Figure 5, it is possible that expert responses will provide similar results for both options. In this case, we'll have to talk with another one of the five senior medical experts to get a clear answer to the issue.

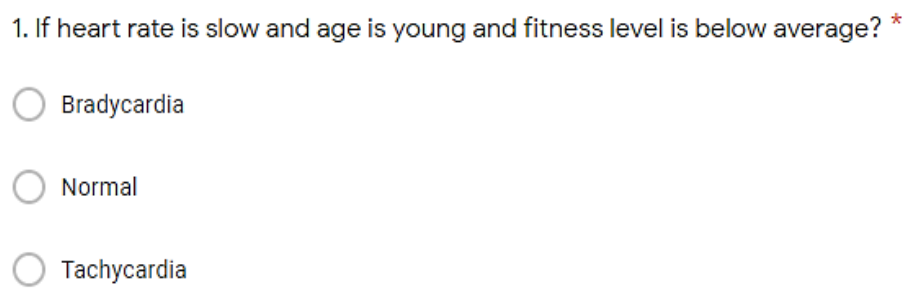

Figure 4. If heart rate is slow and age is young and fitness level is below average? 
7. If heart rate is slow and age is old and fitness level is below average?

5 responses

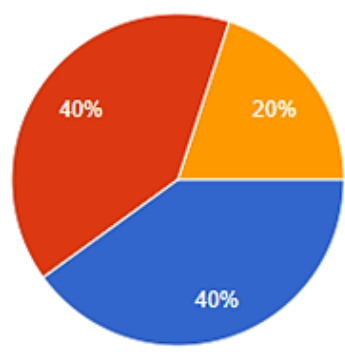

Figure 5. Example answers that have equivalent result

\section{DEVELOPMENT OF FUZZY LOGIC SYSTEMS-HEART RATE CLASSIFICATION}

As previously said, this study used a fuzzy logic approach. In fuzzy logic, there are three steps for classifying heart-rate events, as shown in Figure 6. That is the phases of fuzzification, fuzzy inference, and defuzzification sub-chapters.

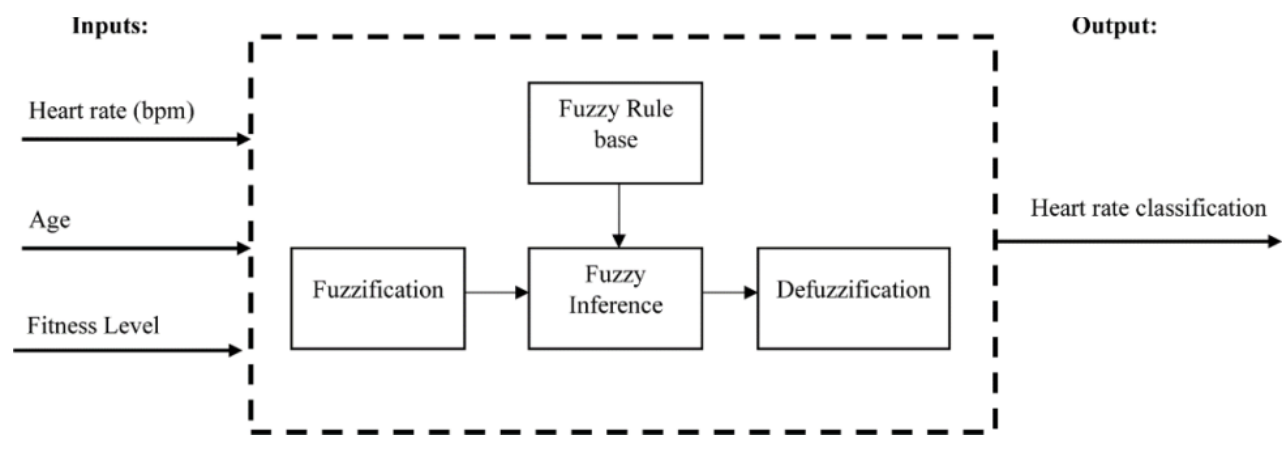

Figure 6. Fuzzy logic system for heart rate calssification

\subsection{Define the fuzzification}

Fuzzification is the first step of fuzzy logic. For all input variables, this step is used to set the fuzzy set. It implies the task of translating a crisp number to a series of fuzzy values. By assigning numerical values to the linguistic component, this is achieved. The input variable for this study would be all of the questions that were triggered in response to heart rate. In this step, the system's input and output variables as shown in Table 1 are specified and translated into linguistic variables. Table 2 demonstrate the input variables with their linguistic variables.

In this study, there are the aforementioned three inputs and one output in this system. The inputs are heart rate, age, and fitness level as shown in Table 2. The output is the heart rate type as shown in Table 1. The type of fuzzy inference system used in this study is the Mamdani inference system. Figure 7 shows the heart rate classification system on the MATLAB programme.

Table 1. Heart rate events

\begin{tabular}{cc}
\hline Types of Heart Rate & Beats Per Minute (BPM) \\
\hline Slow (Bradycardia) & $40-59$ \\
Normal & $60-100$ \\
Fast (Tachycardia) & $101-220$ \\
\hline
\end{tabular}

Table 2. Linguistic variable for all symptoms

\begin{tabular}{cc}
\hline Symptoms & Linguistic Variable \\
\hline Heart Rate & $\{$ Slow, Normal, Fast $\}$ \\
Age & $\{$ Young, Adult, Old $\}$ \\
Fitness Level & $\{$ Below Average, Average, Above Average \\
\hline
\end{tabular}

The heart rate values are the first key input in this study. Three membership functions were modelled for this variable input. That is, there are three types of membership functions: Slow, normal, and fast. This input variable's form of membership functions is also 'gaussmf'. The heart rate variable has a range 
of 40 to 220 . The membership functions for the input variable heart rate are shown in Figure 8 . Note that the same technique applies to all inputs, which will not be discussed in this paper due to page restrictions.

The output variable of the study is heart rate events. Similarly, there are three membership functions for this output variable: Bradycardia, regular, and tachycardia. The class of membership functions in this output variable is lemph\{'trimf'\}. The standards of exercise range from 0 to 4 . Figure 9 shows the membership features for the results variable heart rate type.

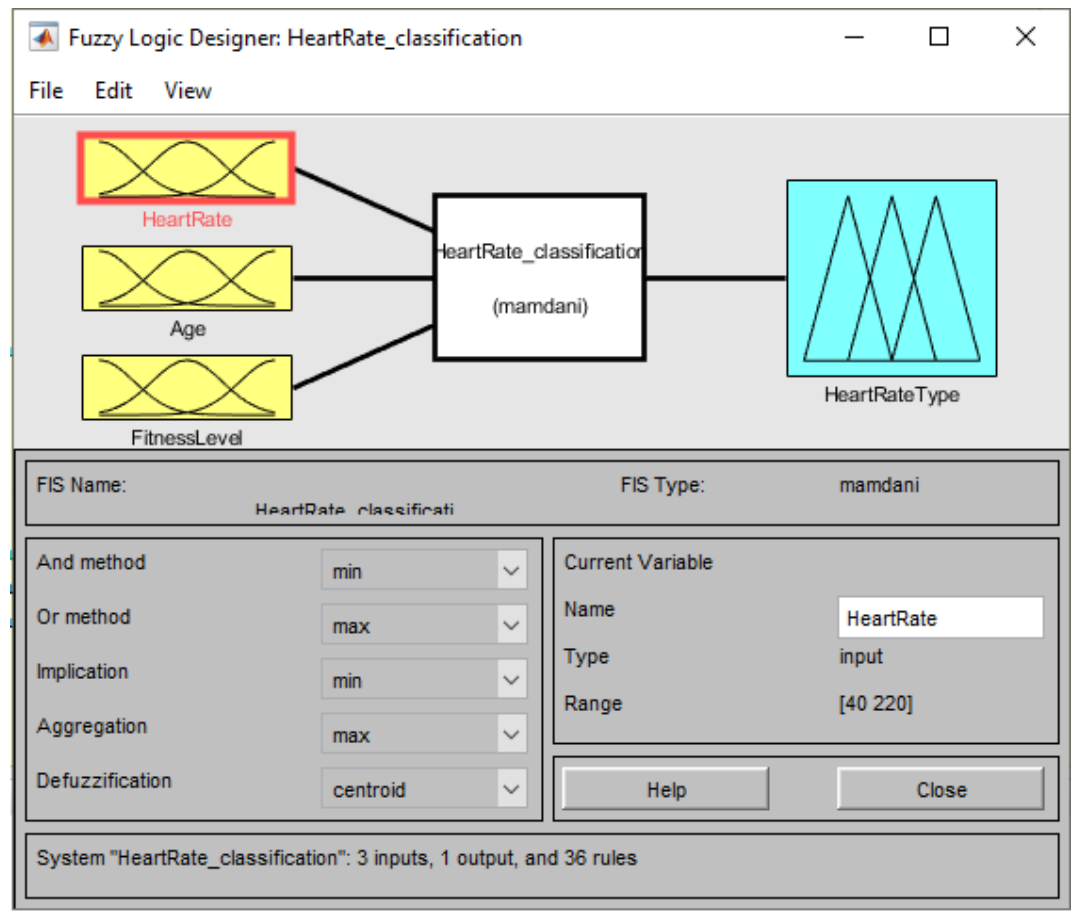

Figure 7. Heart rate classification events

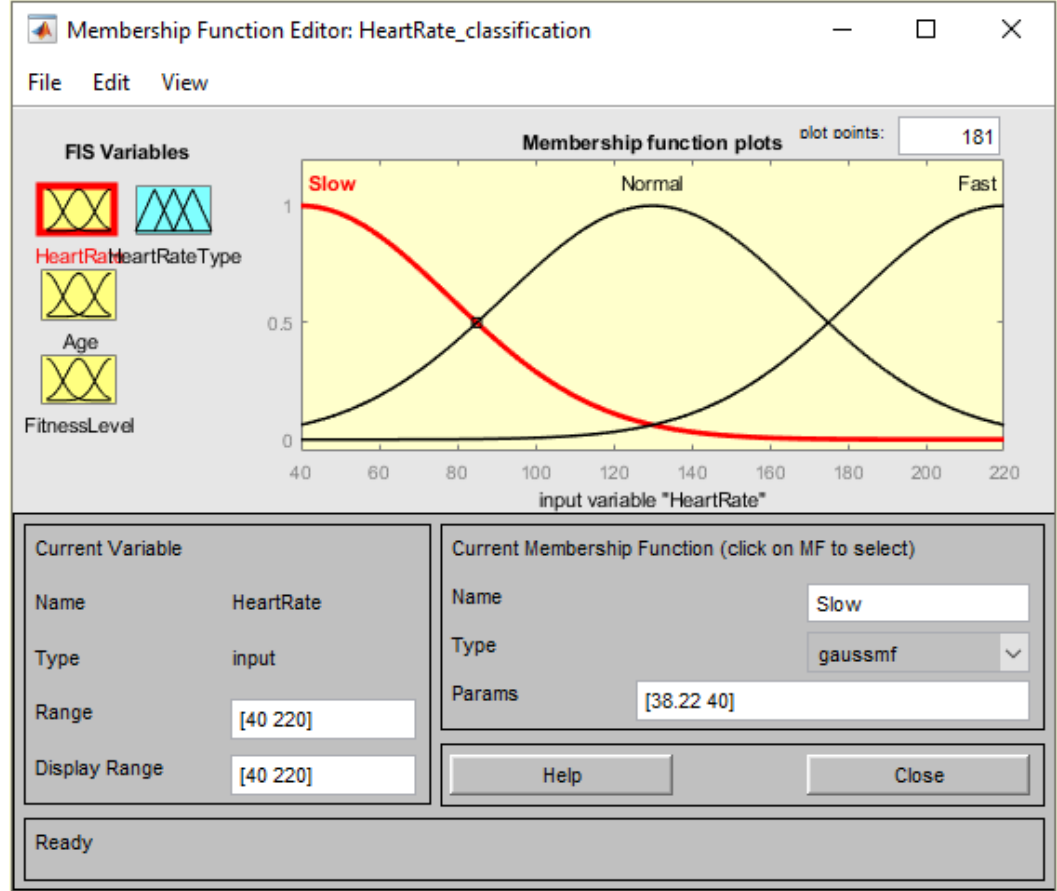

Figure 8. Membership functions for input 'heart rate' 


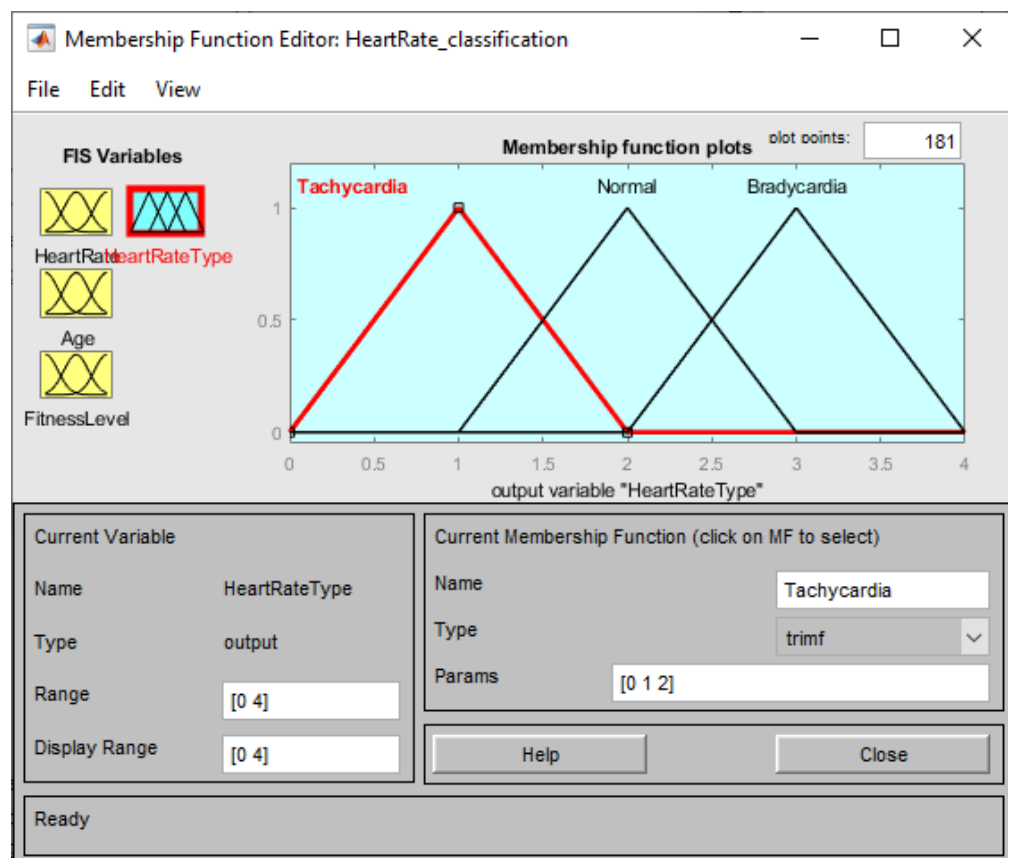

Figure 9. Membership functions for output 'heart rate events'

\subsection{Fuzzy inference system}

Fuzzy inference is the second step of fuzzy logic. The method of mapping the input variables (as previously described) to the output variables is known as fuzzy inference. The heart identification with linguistics component is the study's performance, and it can be classified as bradycardia, normal, or tachycardia. The 'min' operator was used in this study. The conditional part of the law is merged using the min operator. That is, the membership value of the conclusion part is proportional to the lowest membership value of the condition parts. The study's fuzzy rule base is shown in Figure 10. There are 36 rule bases in this analysis. This rule base was created as a result of a survey of medical experts. This rule base includes the 'and' relationship.

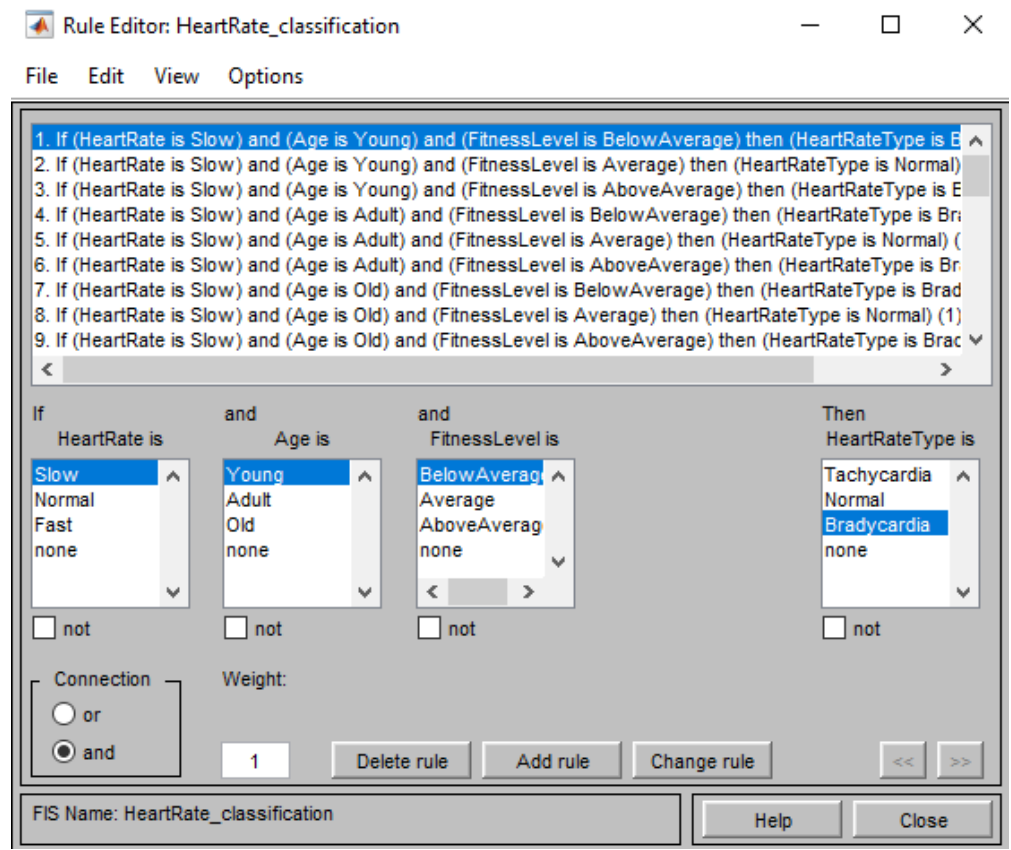

Figure 10. Fuzzy rules base 


\subsection{Defuzzification}

Defuzzification is the third level of fuzzy logic. Three methods can be used in the defuzzification phase: center of area (COA), center of sums (COS), and mean of maxima (MOM). However, only the center of area (COA) technique was used in the analysis. This is because the COA technique will measure the best equilibrium between several output linguistic words. During the defuzzification process, the output is shown using an lemph $\{$ evalfis() $\}$ in MATLAB as can be seen in Figure 11. There are three types of heart rate events: tachycardia, normal, and bradycardia. In this defuzzification step, the users' or patients' findings for this analysis are shown in Figure 11.

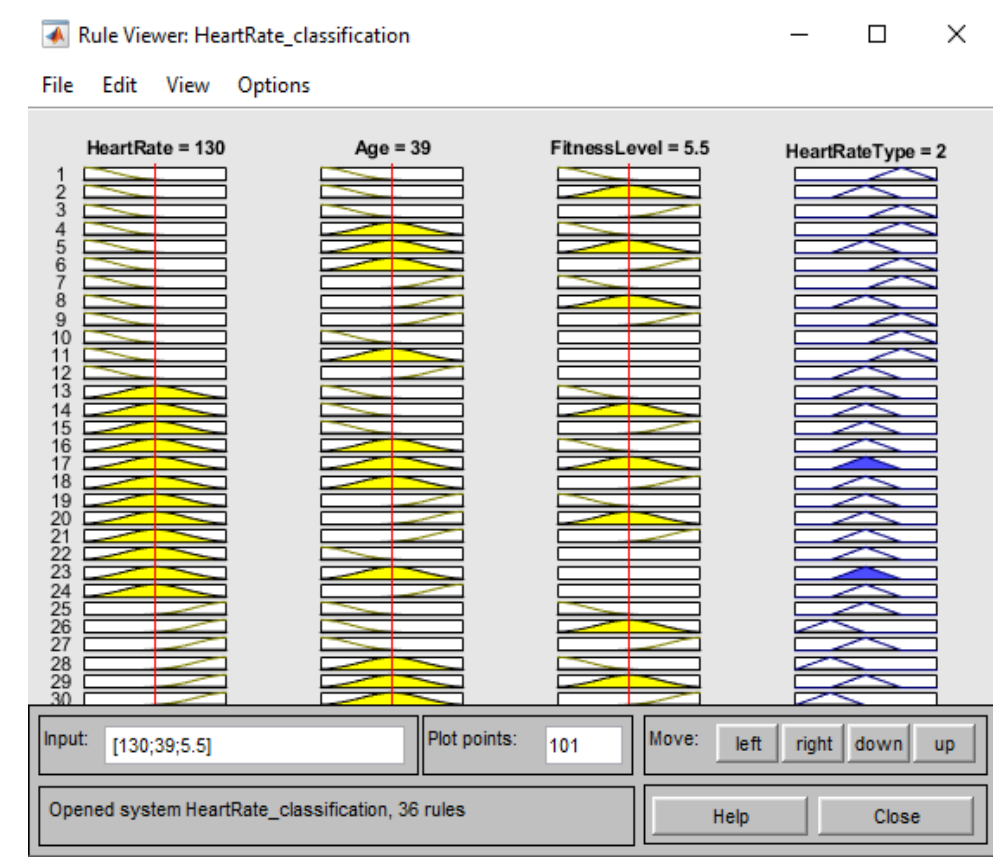

Figure 11. Evaluating of fuzzy inference-heart rate classification events

\section{DEMONSTRATION OF FUZZY LOGIC SYSTEM-GRAPHIC USER INTERFACE}

We will illustrate the proposed design of a fuzzy system in this section. We use the MATLAB graphic user interface (GUI) to view all of the fuzzy components for classifying heart rate events in this example. As a result, the MATLAB GUI has five interfaces: a main menu, a diagnostic page, a research page, a result page, and a background page.

\subsection{GUI-main menu}

Figure 12 depicts the MATLAB GUI's front screen. This page gives you a short rundown of the system. To put it another way, this page can be thought of as a system summary page.

\subsection{GUI-diagnosis}

Figure 13 shows the MATLAB GUI's diagnostic tab. There are five requirements on this diagnosis list. The following information is required: user id, date, heart rate, age, and fitness level. The candidate must complete all five requirements. The participant must press the "SUBMIT" button after completing all the conditions.

\subsection{GUI-results}

The outcome page in the MATLAB GUI as shown in Figure 14. Participants' values from the previous page (diagnosis page) are immediately transferred to this page. Click the "result" button after the participant has verified that the value is the same as the previous page (diagnosis page). The answer will be shown on the page after the participant presses the button. The participants' results are dependent on the diagnosis page. On this tab, the outcome will be shown as bradycardia, regular, or tachycardia. A "fuzzy process" button is also available. A fuzzy logic scheme can be routed to this button. There's even a tab that says "explanation and suggestion". This button can be used to go to the page of explanations and suggestions. 


MainPage
Heart Rate Self-Diagnosis

This system will help you measure your heart rate, in beats per minute

(BPM). Based on your heart rate (HR), age, and fitness level, it will determine your type of heart rate, either bradycardia, normal or tachycardia.

\begin{tabular}{|c|c|}
\hline Analyse & Result \\
$\begin{array}{c}\text { Analyse three } \\
\text { linguistic } \\
\text { variables }\end{array}$ & $\begin{array}{c}\text { Result of } \\
\text { the diagnosis }\end{array}$ \\
\hline
\end{tabular}

Figure 12. Main menu

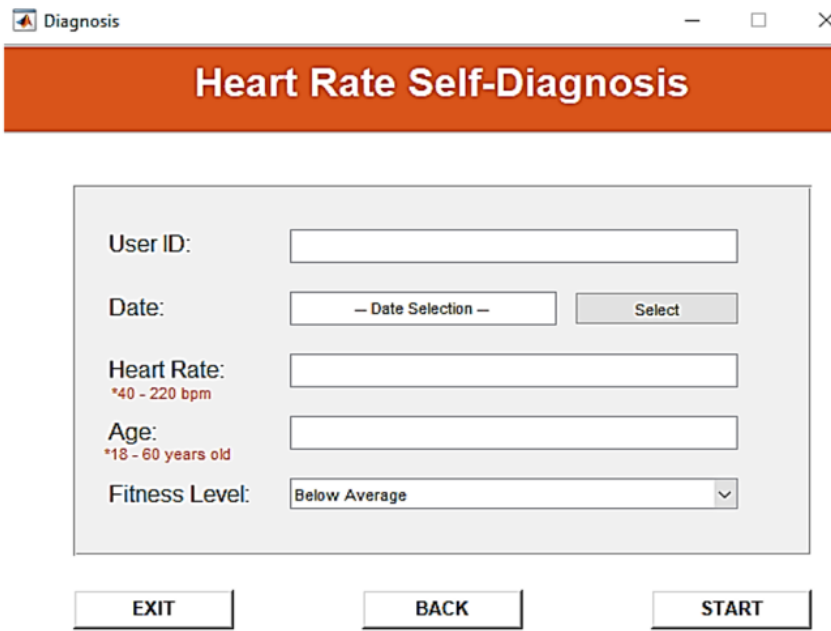

Figure 13. Diagnosis

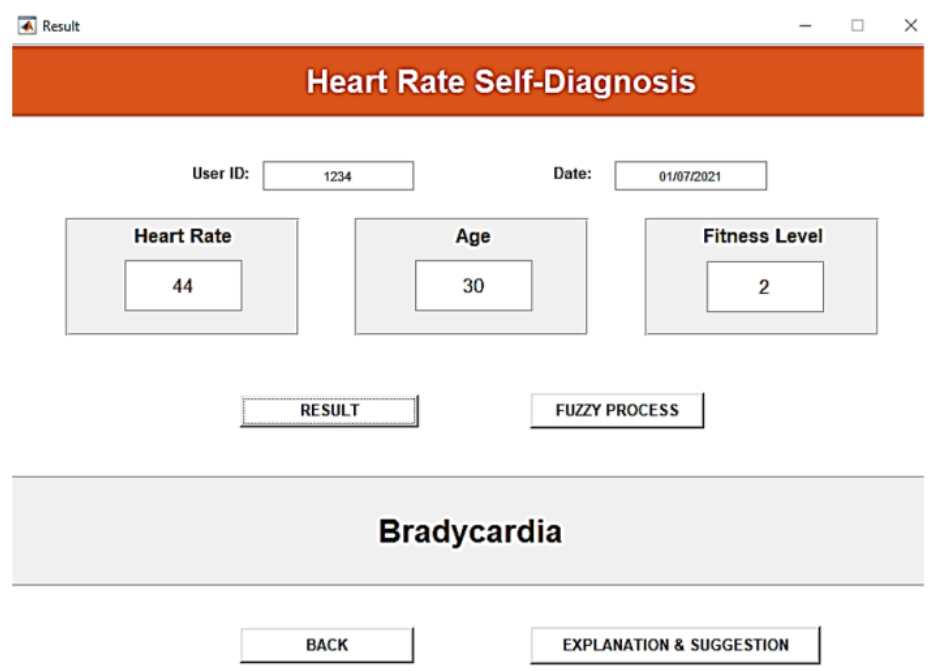

Figure 14. Diagnosis

Int J Artif Intell, Vol. 10, No. 4, December 2021: 1036 - 1047 


\subsection{GUI-explanation and suggestion}

The definition and suggestion tab in the MATLAB GUI as shown in Figure 15(a). There are three different types of heart rate cases. The three types of heart rate incidents are bradycardia, mild, and tachycardia. Each type of heart rate occurrence has a button that takes you to the next tab.

The definition and suggestion for the bradycardia case, for example, as shown in Figure 15(b). This GUI shows basic information about bradycardia (slow heart rate). This GUI also tells the participant whether or not they should see a doctor. The GUI's aim is also to educate the participants about the situation. For instance, describe the symptoms that lead to this heart rate event.

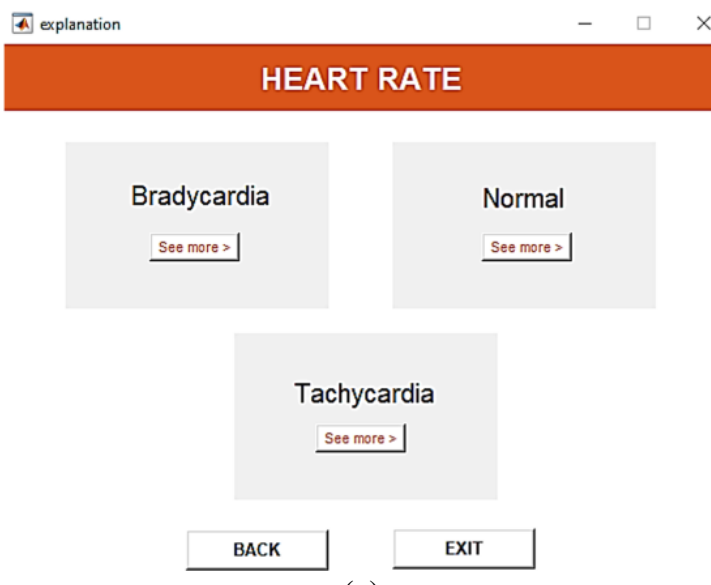

(a)

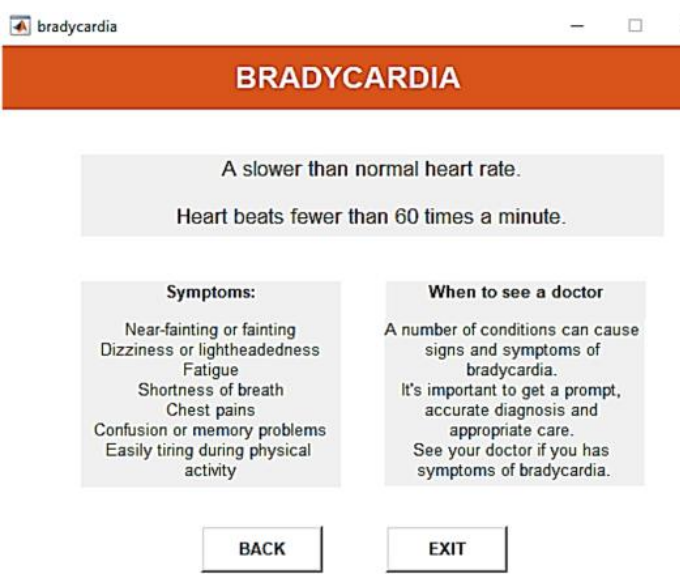

(b)

Figure 15. Explanation and suggestion: (a) main, (b) bradycardia event

\section{PRELIMINARY EVALUATION ON EXPLAINABLE OF THE PROPOSED APPROACH}

In this section, we conduct the preliminary assessment of the proposed fuzzy logic system. The best way to know whether the proposed system is understandable or explainable is by asking the people or the user that used the system [28]. We would like to specifically inquire about the users' understanding and usefulness of the proposed systems. Just ten medical practitioners participate (that knows about heart rate assessment) in the preliminary evaluation.

\subsection{Assessing people perception on understanding of the fuzzy heart rate events system}

This section tends to assess the people perception of the proposed system. Figure 16 shows a bar chart for the first question on the information provided is easy to understand. According to the survey, most participants comprising five out of ten participants equivalent to 50\% agreed that the information provided in the system is easy to understand. This is because the developer uses simple words in the system so that the participants can easily understand, such as the use of linguistic variable of the fuzzy system is easy to grasp. Providentially, none of the participants preferred strongly disagree and disagrees that the information provided in the system is easy to understand. This means that none of the participants did understand the information in the system.

\subsection{Assessing usefulness of the proposed system}

Figure 17 depicts a bar chart illustrating whether it is simple to self-diagnose heart rate using the system rather than visiting a hospital or clinic. According to the poll, the majority of participants agreed that it is simple to do heart rate self-diagnosis across the system rather than going to a hospital or clinic, with seven out of ten participants $(70 \%)$ agreeing. This is due to the fact that by using the system, participants should not have to wait for their turn to be diagnosed. In addition, two out of ten participants, or $20 \%$, firmly accepted that self-diagnosing heart rate across the system is easier than going to a doctor or clinic. This is due to the fact that participants do not have to wait long to learn the outcome of their diagnosis.

Eventually, one out of every ten people, or $1 \%$, said it is simple to do heart rate self-diagnosis through the system rather than going to a doctor or clinic. This is due to the fact that the participant may believe that the system, as well as the hospital or clinic, are simple to diagnose. Fortunately, none of the participants wanted to firmly disagree or disagree that the system's knowledge is easy to understand. This suggests that none of the researchers accepted that using the device to self-diagnose heart rate is impossible. 


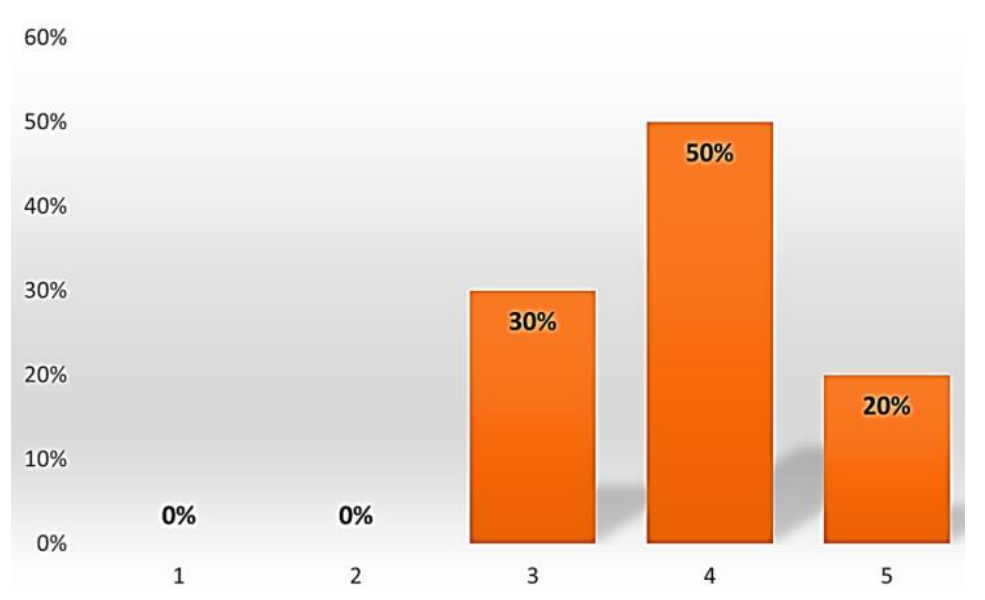

Figure 16. Bar chart on the information provided that is easy to understand

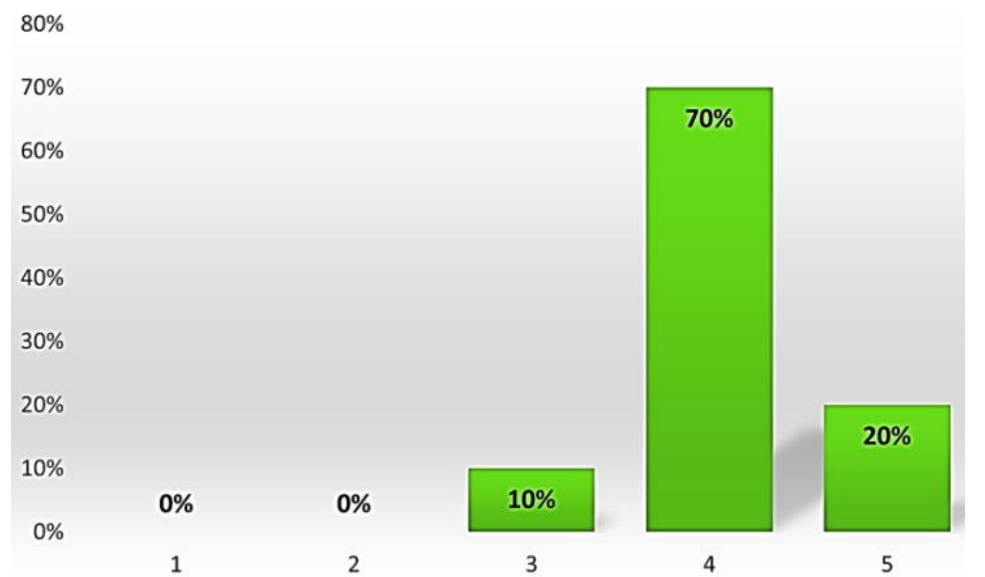

Figure 17. Bar chart on usefulness of the heart rate classification

\section{CONCLUSION}

In conclusion, we have described how to build explainable fuzzy systems for medical applications, specifically heart rate events classification. The method entails three steps: (i) classifying the medical expert's criteria for heart rate signs; (ii) designing an explainable fuzzy logic system for heart rate assessment; and (iii) examining the proposed system with human experts. Despite the fact that this is the initial step in the development, the proposed approach seems to be promising in terms of developing fuzzy systems for heart rate event classification, based on existing information from demonstration and preliminary evaluation. We would like to do further research into more complex medical applications, with a focus on developing a method for designing explainable fuzzy systems. We will also conduct testing with more medical professionals and public customers, resulting in a more understandable fuzzy system for medical applications.

\section{ACKNOWLEDGEMENTS}

We appreciate anyone who took the time to participate in this research. Their responses and detailed comments were very helpful, and they made a significant contribution to this project. This work was supported by the YTR research grant, Universiti Teknologi MARA, Malaysia (Reference No.: 600$\mathrm{RMC} / \mathrm{YTR} / 5 / 3(018 / 2020)$.

\section{REFERENCES}

[1] R. Avram et al., "Real-world heart rate norms in the health eHeart study," npj Digital Medicine, vol. 2, no. 58, 2019, doi: 10.1038/s41746-019-0134-9. 
[2] M. King, J. Kingery, and B. Casey, "Diagnosis and evaluation of heart failure," American Family Physician, vol. 85, no. 12, pp. 1161-1168, 2012.

[3] American Heart Association, "All about heart rate (Pulse)," Heart.Org. Accessed 1 June $2021<$ https://www.heart.org/en/health-topics/high-blood-pressure/the-facts-about-high-blood-pressure/all-about-heartrate-pulse>.

[4] M. Galetakis and A. Vasileiou, "Applications of fuzzy inference systems in mineral industry-overview," Expert System Software. pp. 211-226, 2013.

[5] D. M. Sherer, M. Dalloul, N. Pierre, and O. Abulafia, "Intrapartum repetitive maternal heart rate deceleration pattern simulating nonreassuring fetal status," American Journal of Perinatology, vol. 22, no. 3, pp. 165-167, 2005, doi: $10.1055 / \mathrm{s}-2005-864852$.

[6] A. Garrido, "A brief history of fuzzy logic," Broad Research in Artificial Intelligence and Neuroscience (BRAIN), vol. 3, no. 1, pp. 71-77, 2012.

[7] J. M. Mendel, Uncertain rule-based fuzzy logic systems: Introduction and new directions, Prentice Hall PTR, 2001.

[8] T. R. Razak, J. M. Garibaldi, C. Wagner, A. Pourabdollah and D. Soria, "Toward a framework for capturing interpretability of hierarchical fuzzy systems-a participatory design approach," in IEEE Transactions on Fuzzy Systems, vol. 29, no. 5, pp. 1160-1172, May 2021, doi: 10.1109/TFUZZ.2020.2969901.

[9] T. R. B. Razak, R. A. Wahab and M. H. Ramli, "Dengue notification system using fuzzy logic," Int. Conf. on Computer, Control, Informatics and Its Applications, 2013, pp. 231-235, doi: 10.1109/IC3INA.2013.6819179.

[10] F. Shaffer and J. P. Ginsberg, "An overview of heart rate variability metrics and norms," Frontiers in Public Health, 2017, doi: 10.3389/fpubh.2017.00258.

[11] M. R. Helton, "Diagnosis and management of common types of supraventricular tachycardia," American Family Physician, vol. 92, no. 9, pp. 793-802, 2015.

[12] A. U. Mahtani and D. G. Nair, "Supraventricular Tachycardia," The Medical Clinics of North America, vol. 103, no. 5, pp. 863-879, 2019, doi: 10.1016/j.mcna.2019.05.007.

[13] K. C. Luk, J. E. Ball, and A. Sharma, "A study of optimal model lag and spatial inputs to artificial neural network for rainfall forecasting," Journal of Hydrology, vol. 227, no. 1-4, pp. 56-65, Jan. 2000, doi: 10.1016/S00221694(99)00165-1.

[14] D. A. Pisner and D. M. Schnyer, "Support vector machine," in Machine Learning: Methods and Applications to Brain Disorders, 2020, pp. 101-121, doi: 10.1016/B978-0-12-815739-8.00006-7.

[15] H. Sharma and S. Kumar, "A survey on decision tree algorithms of classification in data mining," International Journal of Science and Research (IJSR),vol. 5, no. 4, pp. 2094-2097, 2016, doi: 10.21275/v5i4.nov162954.

[16] R. Moonjun, D. P. Shrestha, and V. G. Jetten, "Fuzzy logic for fine-scale soil mapping: a case study in Thailand," Catena, vol. 190, Jul. 2020, doi: 10.1016/j.catena.2020.104456.

[17] T. R. Razak, J. M. Garibaldi and C. Wagner, "An improved complexity measure in hierarchical fuzzy systems," IEEE International Conference on Fuzzy Systems (FUZZ-IEEE), 2020, pp. 1-8, doi: 10.1109/FUZZ48607.2020.9177736.

[18] M. Phillips et al., "Prediction of breast cancer using volatile biomarkers in the breath," Breast Cancer Research and Treatment, vol. 99 , no. 1, pp. 19-21, Sep. 2006, doi: 10.1007/s10549-006-9176-1.

[19] N. Bhatla and K. Jyoti, "A novel approach for heart disease diagnosis using data mining and fuzzy logic," International Journal of Computer Applications, vol. 54, no. 17, pp. 16-21, 2012, doi: 10.5120/8658-2498.

[20] T. R. Razak et al., "Towards Designing a hierarchical fuzzy system for early diagnosis of heart disease," Journal of Computing Research and Innovation (JCRINN), vol. 4, no. 2, pp. 31-41, Nov. 2019.

[21] J. Schneider, N. Bitterlich, H.-G. Velcovsky, H. Morr, N. Katz, and E. Eigenbrodt, "Fuzzy logic-based tumormarker profiles improved sensitivity in the diagnosis of lung cancer," International Journal of Clinical Oncology, vol. 7, no. 3, pp. 145-51, Jun. 2002, doi: 10.1007/s101470200021.

[22] M. Phillips et al., "Prediction of lung cancer using volatile biomarkers in breath," Cancer Biomarkers: section A of Disease Markers, vol. 3, no. 2, pp. 95-109, Jan. 2007, doi: 10.3233/cbm-2007-3204..

[23] A. M. Badawi, A. S. Derbala, and A.-B. M. Youssef, "Fuzzy logic algorithm for quantitative tissue characterization of diffuse liver diseases from ultrasound images," International Journal of Medical Informatics, vol. 55, no. 2, pp. 135-147, Aug. 1999, doi: 10.1016/S1386-5056(99)00010-6.

[24] K. Polat, S. Şahan, H. Kodaz, and S. Güneş, "Breast cancer and liver disorders classification using artificial immune recognition system (AIRS) with performance evaluation by fuzzy resource allocation mechanism," Expert Systems with Applications, vol. 32, no. 1, pp. 172-183, Jan. 2007, doi: 10.1016/j.eswa.2005.11.024.

[25] M. F. Ganji and M. S. Abadeh, "A fuzzy classification system based on Ant colony optimization for diabetes disease diagnosis," Expert Systems with App., vol. 38, no. 12, pp. 14650-14659, Nov. 2011, doi: 10.1016/j.eswa.2011.05.018.

[26] C. Lee and M. Wang, "A fuzzy expert system for diabetes decision support application," in IEEE Trans. on Systems, Man, and Cybernetics, Part B (Cybernetics), vol. 41, no. 1, pp. 139-153, 2011, doi: 10.1109/TSMCB.2010.2048899.

[27] C. Wagner, M. Smith, K. Wallace and A. Pourabdollah, "Generating uncertain fuzzy logic rules from surveys: capturing subjective relationships between variables from human experts," 2015 IEEE International Conference on Systems, Man, and Cybernetics, 2015, pp. 2033-2038, doi: 10.1109/SMC.2015.355.

[28] T. R. Razak, J. M. Garibaldi, C. Wagner, A. Pourabdollah and D. Soria, "Interpretability and complexity of design in the creation of fuzzy logic systems-a user study," 2018 IEEE Symposium Series on Computational Intelligence (SSCI), 2018, pp. 420-426, doi: 10.1109/SSCI.2018.8628924. 